\title{
A new enigmatic species of broad-nosed weevil endemic to Brazil and its phylogenetic placement within the tribe Naupactini (Coleoptera: Curculionidae: Entiminae)
}

\author{
María Guadalupe del Río ${ }^{1,2}$ \& Analía Alicia Lanteri ${ }^{1,3}$ \\ 1 Universidad Nacional de La Plata (UNLP), Facultad de Ciencias Naturales y Museo (FCNyM), División Entomología. \\ La Plata, Buenos Aires, Argentina. Consejo Nacional de Investigaciones Científicas y Técnicas (CONICET). \\ 2 ORCID: http://orcid.org/0000-0001-9865-6099. E-mail: gdelrio@fcnym.unlp.edu.ar \\ 3 ORCID: http://orcid.org/0000-0002-8115-7282. E-mail: alanteri@fcnym.unlp.edu.ar
}

\begin{abstract}
A new species of Naupactini (Curculionidae: Entiminae) endemic to Brazil, Espírito Santo and Minas Gerais states, is herein described. It resembles the monotypic genus Hadropus Schoenherr in its general appearance, particularly in the shape of the elytra, and the color of the vestiture, but the results of a cladistics analysis herein conducted suggest that it belongs to the genus Stenocyphus Marshall. This genus ranges in São Paulo, Rio de Janeiro and Espírito Santo, mainly in the Atlantic forest of Brazil, and includes three other species. Stenocyphus costae sp. nov., distinguishes from the remaining species of Stenocyphus by the green iridescent scaly vestiture, having long stiff setae on the two pairs of elytral tubercles; the more slender rostrum; the shorter antennae; the convex disc of the pronotum; and the shorter and broader elytra. This paper includes a cladogram of the Naupactini genera showing the phylogenetic position of the new species, its complete description, photographs of male and female habitus, line drawings of genitalia of both sexes, and a key of the Stenocyphus species.
\end{abstract}

Key-Words. Hadropus; Neotropical region; South America; Stenocyphus.

\section{INTRODUCTION}

The Neotropical genera Hadropus Schoenherr, 1826 and Stenocyphus Marshall, 1922 (Curculionidae, Entiminae, Naupactini) share several morphological characters, such as the presence of elytral tubercles and the disc of the elytra elevated towards the beginning of the declivity (humped elytra); the vestiture composed of scales and coarse setae; the tibiae lacking rows of denticles along the inner margin or with minute denticles; and the corbels of the metatibiae well-developed, covered with scales or scale-like setae. Hadropus is a monotypic genus that ranges in Paraguay and Brazil, mainly in the Chacoan and Parana subregions (del Río \& Lanteri, 2011), and Stenocyphus is endemic to Brazil, and is mainly distributed along the Atlantic forest, from São Paulo to Espírito Santo (del Río \& Lanteri, 2013). Mixed in the series of Hadropus albiceris Germar, we found some specimens which are similar to this species in the characters of the vestiture and shape of the elytra, but differentiate in some diagnostic characters at generic level, such as those of the rostrum and shape of the spermatheca (see del Río
\& Lanteri, 2011). We concluded that these specimens correspond to a new species, and in order to test its generic position we undertook a cladistics analysis based on morphological characters.

The objectives of this contribution are to describe the new species, to illustrate its diagnostic characters, and to analyze its phylogenetic position within the tree of the Naupactini, to decide about to its correct generic placement.

\section{MATERIAL AND METHODS}

Specimens of the new species are very scarce in entomological collections, and they would be rare in nature. This study was based upon the examination of specimens borrowed from the following institutions: AMNH: American Museum of Natural History, New York, USA. Lee Herman; DZUP: Departamento de Zoologia, Universidade Federal do Parana, Curitiba, PR, Brazil. Germano Rosado-Neto; MLPC: Museo de La Plata, Buenos Aires, Argentina. María Guadalupe del Río; MZSP: Museu de Zoologia da Universidade do São Paulo, SP, Brazil. Sergio Vanin. 
Dissections of female and male genitalia were made according to standard entomological techniques. Characters of the genitalia were drawn using a camera lucida adapted to a stereoscopic microscope Nikon SMZ800. Measurements were taken with an ocular micrometer attached to this microscope. The abbreviations used in the description are as follows: $L=$ maximum length; $W=$ maximum width; $W R=$ width of rostrum measured across apex (excluding scrobes); WF = width of forehead between anterior margins of eyes.

The key for the species of Stenocyphus was expanded from that of del Río \& Lanteri (2013).

\section{Phylogenetic analysis}

The morphological characters and character states used for this phylogenetic analysis were listed in Lanteri \& del Río (2017) (Table S1). The list consisted of 100 discrete morphological characters of the adults, including 78 from the external morphology and 22 from the female and male genitalia.

A data matrix herein analyzed includes 76 terminal taxa (72 for the ingroup plus four outgroups) and 100 morphological characters (Table S2). Character states unknown were scored with "?" and treated as missing data (Maddison, 1993). All characters were treated as non-additive and were analyzed under equal and implied weights $(K=45)$.

Searches were conducted using the "traditional search" algorithm of TNT (Goloboff \& Catalano, 2016), with 300 random addition sequences, Tree Bisection and Reconnection (TBR) branch swapping, holding 20 trees during each replication. Clade stability was evaluated with 1000 replication Bootstrap (BT) (Felseinstein, 1985). We provide the total length $(\mathrm{L})$, the consistency index $(\mathrm{Cl})$ (Kluge \& Farris, 1969) and the retention index (RI) (Farris, 1989) of the most parsimonious trees (MP tree), calculated excluding the uninformative characters. Since more than one most parsimonious tree was obtained, we calculated a strict consensus tree among them (Goloboff \& Farris, 2001).

\section{RESULTS}

\section{Cladistic analysis}

The heuristic search under equal weights yielded 35 equally parsimonious trees $(\mathrm{L}=816$ steps, $\mathrm{Cl}=0.21$, $\mathrm{RI}=0.54)$. In the consensus tree (Fig. 1), same as in the analysis under implied weights $(k=45)$, the new species is recovered in a clade including the species of Stenocyphus and Neoerycideus Hustache, 1938, mainly supported by the elytral disc ascending towards the beginning of the declivity (= humped) (char. 53.1) and the denticles of the inner margin of tibiae absent or minute (char. 70.0). The new species form a clade with the other three species of Stenocyphus (BT: 36), mainly supported by the presence of lateral rostral carinae (char. 11.1); gular angle slightly obtuse (char. 27.1); disc of pronotum with irregular foveae or large punctures (char. 48.1); elytra with tubercles on disc and declivity (char. 57.1); apex of median lobe of aedeagus rounded (char. 96.2) and endophallus of penis with flagellum (char. 98.1).

The three species of Stenocyphus (BT: 66) differentiated from the new species because they share the following characters: postocular constriction of head strong (char. 26.1); pronotum with a pair of lateral, longitudinal impressions (char. 42.1); uneven intervals of elytra (except suture) strongly convex, even intervals flat (char. 59.2); corbel at metatibial apex broad, squamose (char. 71.0); spermathecal corpus subcylindrical, slender (char. 89.0). Within this clade, S. bituberosus (Gyllenhal, 1833 ) is sister to S. sextuberosus del Río \& Lanteri, 2013 (BT: 81).

The new species is not related to Hadropus, which is included in another clade of Naupactini where members also have humped elytra (see Fig. 1).

\section{Systematics}

\section{Stenocyphus costae sp. nov. (Figs. 2, 3)}

Type material: Holotype 으 (DZUP): $10 \mathrm{~mm}$ long, with labels as follows: "Sta. Tereza-ES/ Brasil 07-XII-1964, C. Elias leg." (dissected with genitalia in vial with glycerin). Paratypes: Same data as holotype (19 dissected with genitalia in vial with glycerin, MLP). Brazil, Espírito Santo, Tijuco Preto, Oct. 1948, A. Maller coll., Frank Johnson Donor (19, AMNH); Minas Gerais, Serra do Caraça, 27-XI a 05-XII-1972, Exp. Mus. Zool. (10' dissected with genitalia in vial with glycerin, MZSP).

Diagnosis: Stenocyphus costae sp. nov., is distinguished from the remaining species of Stenocyphus by the color of the scaly vestiture, iridescent green instead of cream or tan, and opaque; the longer and more slender rostrum; the shorter antennae, with scape almost reaching half of eyes, and funicular articles 3 to 7, 1.5-2.0x as long as wide; the strongly convex pronotum; the shorter elytra, with bisinuate anterior margin not projecting towards pronotum; the presence of two pairs of elytral tubercles covered with brown, stiff, long scale-like setae; the scuteIlum lacking scales; the narrow corbel of the metatibiae, covered with scale-like setae; the slender intercoxal portion of abdomen; the slightly shorter apodeme of sternite VIII and ovipositor; and the longer spermathecal duct.

Description: Species medium sized (female 8.7-10.0 mm; male $7.5 \mathrm{~mm}$ ). Integument visible reddish-brown. Vestiture iridescent greenish, consisting on contiguous, small, subcircular, striate scales and suberect, coarse scale-like setae, stiff and long on the elytral tubercles (Figs. 2A-E).

Head (Figs. 2A-B): Rostrum slender, distinctly longer than wide at apex (L/WR: 1.35-1.55), sides convergent to- 


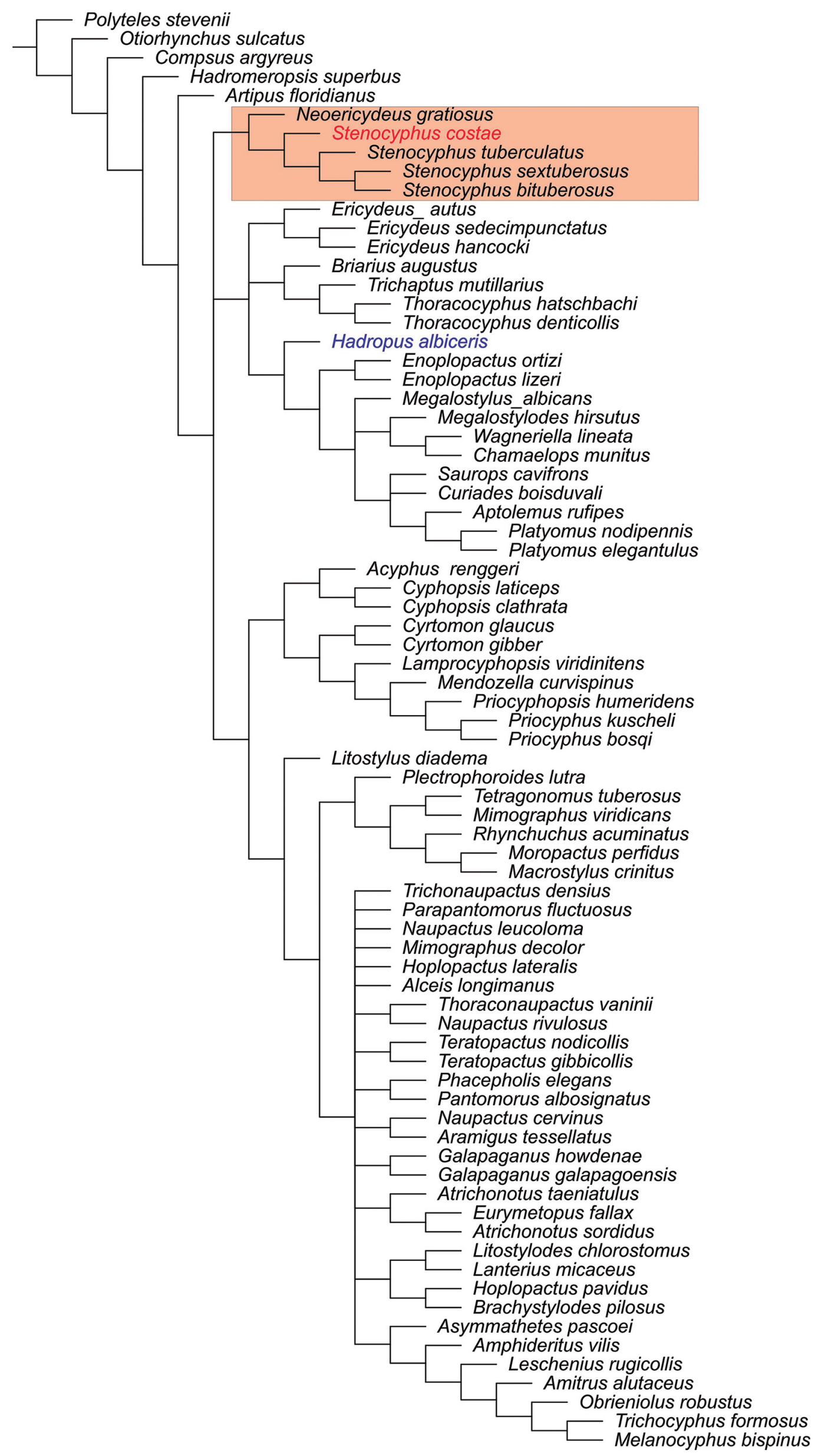

Figure 1. Strict consensus cladogram of $35 \mathrm{MP}$ trees, showing the phylogenetic position of Stenocyphus costae sp. nov. 
wards apex (WF/WR: about 1.5); dorsum flat; lateral carinae present; epistome strongly impressed, covered with small, scattered, subcircular scales; median groove linear, slightly exceeding hind margin of eyes; scrobes slightly curved downwards and directed towards base of eyes; gular angle about $120^{\circ}$.

Mouthparts: Mandibles covered with creamy scatter scales and coarse yellowish setae on external face; premen- tum subpentagonal, with external surface lacking setae. Eyes slightly drop-shaped, moderately convex; preocular impression triangular, elongate; postocular constriction indistinct. Forehead flat; vertex slightly convex. Antennae (Fig. 3A) short, $1 / 4$ of total body length, slightly exceeding pronotal margin, with setose scape and funicle; scape reaching half of eyes, about half length of funicle; funicular article 2, $2 \times$ as long as article 1 , articles 3 to $7,1.5-2 \times$ longer than wide; club acuminate, oval (L/W: 1.95-2.15).
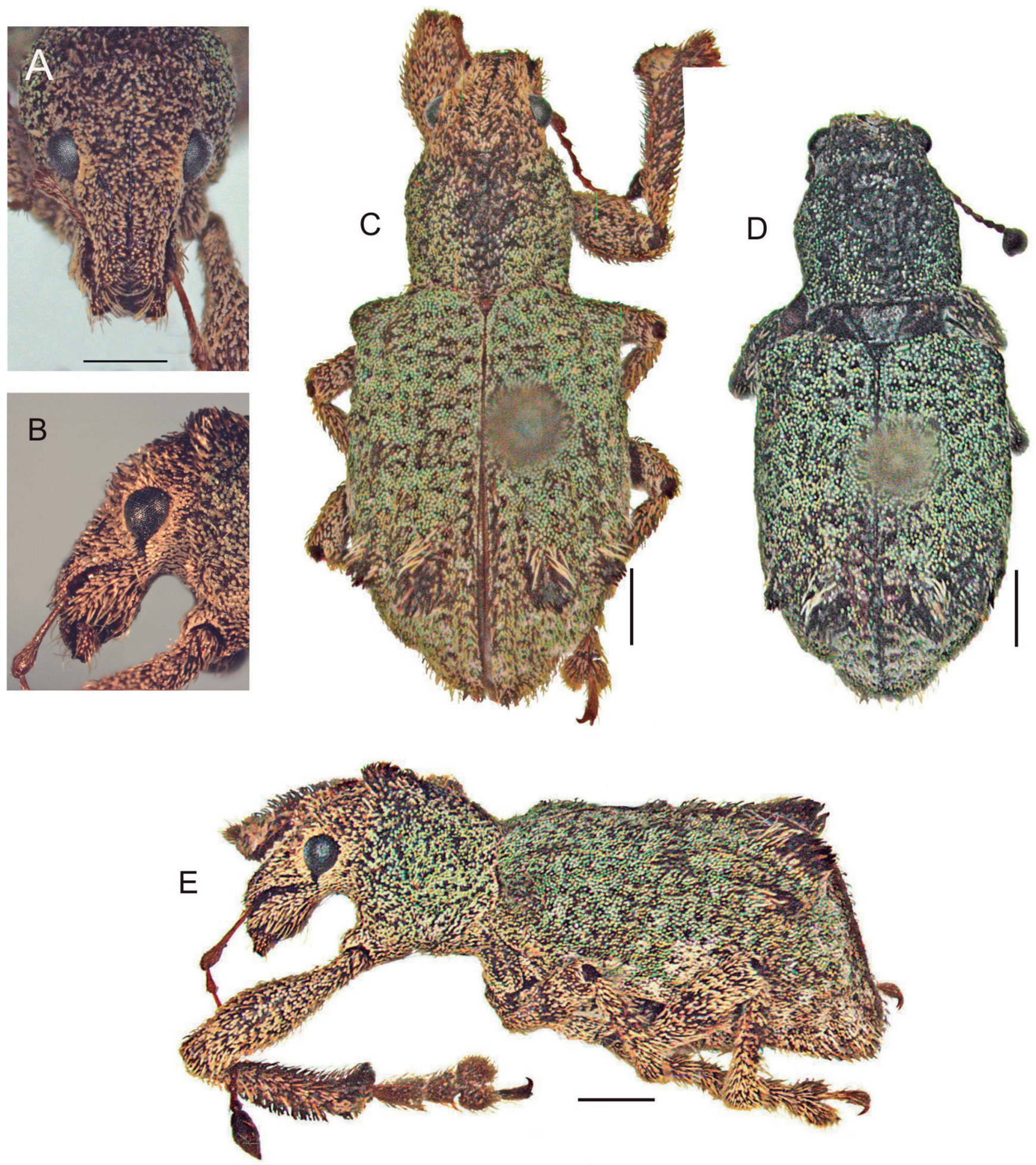

Figure 2. Stenocyphus costae sp. nov. Paratype female: (A) head, frontal; (B) head, lateral; (C) habitus dorsal; (E) habitus lateral. Paratype male: (D) habitus dorsal. Scale bars: $1 \mathrm{~mm}$. 
Pronotum (Figs. 2C-D): As long as wide to slightly transversal (L/W: 1.05-1.25), sides divergent towards anterior $1 / 3$ and almost subparallel on posterior $2 / 3$, maximum width on anterior $1 / 3$; disc slightly convex and elevated from base to apex, disc with irregular foveae and not well-defined median groove; anterior margin slightly curved anteriad; base bisinuate, with postero-lateral angles projected backwards.

Scutellum: Subtriangular, devoid of scales (Figs. 2C-D).

Elytra: Oval, short (L/W: 1.35-1.40), moderately high in lateral view, strongly ascending towards beginning of declivity (= humped) (Fig. 2E); base strongly bisinuate, not projecting towards pronotum; humeri strongly prominent, postero-laterally projected, without tooth; apex entire, subacute; apical declivity moderately abrupt (Figs. 2C-D); subapical callus distinct; punctures of striae medium sized (slightly narrower than intervals) with one oval scale on bottom; intervals wavy (with transversal undulations, or impressions and elevations); two pairs of tubercles present at beginning of declivity, the largest on interval 3, and the smallest on interval 5. Metathoracic wings well-developed.

Legs: Procoxae contiguous, $2 \times$ closer to anterior margin than to posterior margin of prosternum; protibiae strongly curved onwards, without denticles on inner face, and with erect thick setae on inner and outer face; pro- and mesotibiae with mucro and metatibiae without mucro; corbels of metatibiae well developed, covered with scale-like setae; dorsal comb slightly shorter than distal comb; tarsomere 2 as long as wide; tarsal claws free.
Abdomen (Fig. 3B): Intercoxal portion 1.30-1.35x broader than cavities of metacoxae; ventrite 2 about $1.4 \times$ as long as ventrites $3+4$ (1.2× in males).

Female terminalia: Sternite VIII (Fig. 3C) subrhomboidal, slightly sclerotized, with V-shaped sclerotization and long apical setae; apodeme about $1.5 \times$ as long as plate of sternite. Ovipositor (Fig. 3D) about $0.6 \times$ as long as ventrites $1-5$, slightly curved on lateral view; coxites slightly sclerotized, with short setae; baculi subparallel; styli well- developed, directed backwards. Spermatheca (Fig. 3E) about $1 / 8$ as long as ventrites 1-5, somewhat subglobose, with short collum, well- developed ramus and long cornu; spermathecal gland $1.5 \times$ as long as spermatheca; spermathecal duct fine, moderately sclerotized, very long (almost twice as long as ovipositor, about $10 \times$ as long as spermatheca).

Male genitalia: Penis as long as ventrites 1-5; tube with rounded apex (Fig. 3F), slightly longer than apodemes, fairly curved in lateral view (Fig. 3G), subterminal narrow ostium; endophallus with pair of sclerotized elongate sclerites, and long, curled flagellum (Fig. 3G).

Etymology: The species is named in honor of Dr. Cleide Costa, the outstanding Brazilian Coleoptera specialist.

Geographic distribution: Stenocyphus costae sp. nov., is known only for Espírito Santo and Minas Gerais states in Brazil. It is distributed on hilly areas of central-eastern Espírito Santo state, Santa Teresa, and the Serra do Caraça in Minas Gerais, which are protected areas of Atlantic forest.

Remarks: Stenocyphus costae sp. nov., is the sister species of the remaining three species of Stenocyphus.
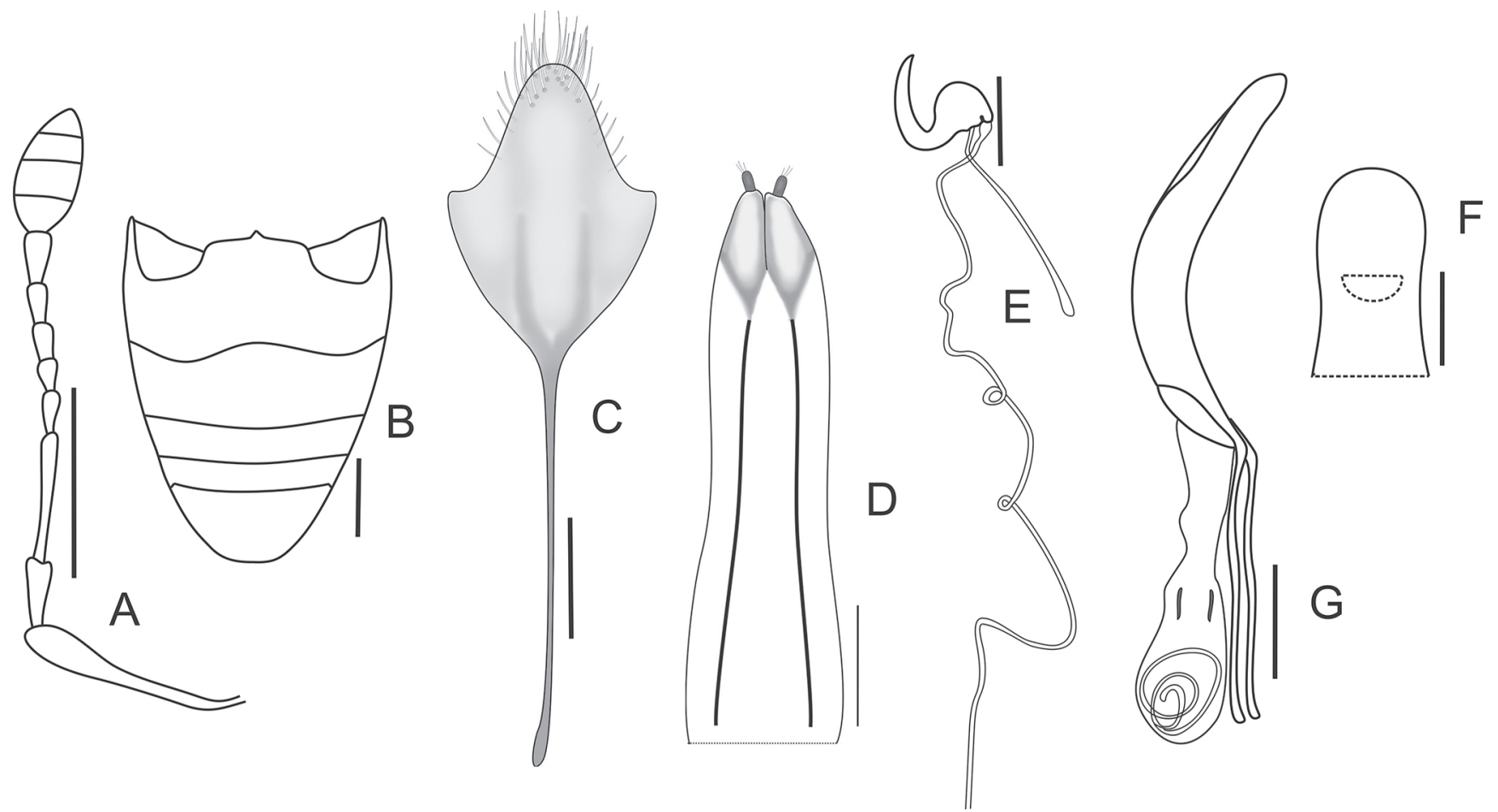

Figure 3. Stenocyphus costae sp. nov. Female: (A) left antenna; (B) ventrites 1-5; (C) sternite VIII; (D) ovipositor, ventral; (E) spermathecae with spermathecal duct. Male: (F) aedeagus, terminal portion of tube, ventral; $(G)$ aedeagus, lateral. Scale bars: $A-B=1 \mathrm{~mm} ; C-G=0.5 \mathrm{~mm}$. 


\section{Key to species of Stenocyphus Marshall}

1. Scaly vestiture iridescent green; scape reaching half of eyes; elytral tubercles covered with brown, stiff, long scale-like setae (Fig. 2).........

Stenocyphus costae sp. nov.

1. Scaly vestiture cream or tan, and opaque; scape exceeding posterior margin of eyes; elytral tubercles not covered with brown, stiff, long scale-like setae

2. Elytral disc with one pair of large conical tubercles, slightly directed backwards, on posterior two thirds of interval 5, near declivity; scutellum suboval; protibiae without mucro and denticles; ovipositor with coarse setae along external sides of apical $2 / 3$ of baculi (see del Río \& Lanteri, 2013, figs. 1, 4) S. bituberosus

2. Elytral disc with more than one pair of tubercles; scutellum subtriangular; protibiae with mucro and denticles; ovipositor without coarse setae along sides of baculi.

3. Elytral disc with three pairs of tubercles, two on interval 3 and one on interval 5; the largest pair of tubercles slightly directed backwards, placed on posterior $2 / 3$ of interval 3 , and followed by a small one; tubercle on interval 5, large but rounded and placed near declivity; penis flattenned towards apex in lateral view (see del Río \& Lanteri, 2013, figs. 2, 5) S. sextuberosus

3'. Elytral disc with three series of conical tubercles along intervals 3,5 and 7 , from base to apex, with the largest tubercles placed near declivity of interval 3; penis not flattened towards apex in lateral view (see del Río \& Lanteri, 2013, figs. 3, 6) S. tuberculatus

\section{DISCUSSION}

The new species is very similar in appearance to that of the genus Hadropus, but besides this superficial similarity it is not related to this genus (see results of the cladistic analysis). In the single species of Hadropus the rostrum is much shorter and wider, and lacks lateral carina; the epistome is more differentiated from the post-rostrum; the spermatheca has a particular shape (without collum, with broad and prominent ramus); and the penis lacks flagellum. All these characters are important to separate genera in the tribe Naupactini.

On the contrary, Stenocyphus costae sp. nov., shares most synapomorphies of Stenocyphus (see results of the cladistic analysis) and has a particular combination of features that allow distinction from the other species of this genus: e.g., scape not reaching hind margin of eye (char. 31.0); club oval, very short (length/width 2.0 to $2.25 \times$ ) (char. 37.0); pronotum convex (char. 44.1), with postero-lateral angles projected (char. 45.1); procoxae twice as close to anterior margin than to posterior margin of prosternum (char. 65.2); and apodeme of sternite VIII less than $2 \times$ as long as plate (char. 80.0).

With the inclusion of the new species in Stenocyphus the distribution of this genus extends western to the state of Minas Gerais. Consequently, this genus does not occur only in the Atlantic Forest (oriental slopes of the coastal hills of Brazil, characterized by a pluvial forest of trees of 30-40 meters, with a lower stratum rich in palms, lianas and epiphytes), but also in the transition area with the Cerrado (largest savanna forest, with open forest having low trees, shrubs, a stratum of herbs rich in Poaceae and Fabaceae). The Serra de Caraça (Minas Gerais), where the species S. costae sp. nov., coexists with Hadropus albiceris, is a unique area of great biological diversity, as it covers a variety of habitats, including montane forest, cloud forest, shrubby montane savannas and high-altitude grasslands. It contains species endemic to the Cerrado (particularly those restricted to campos rupestres in the Espinhaço mountain range), as well as Atlantic Forest endemics. This is an area of high conservation priority in Minas Gerais, where the flora and fauna are currently threatened of extinction (Costa et al., 1998).

It would be very important to collect more extensively in this area of Brazil to find out the accurate distribution of Stenocyphus and its possible intraspecific variation.

\section{ACKNOWLEDGMENTS}

We thank all the specialists and curators that loaned us specimens for study, and/or facilitate the examination of the material; to Lic. Emilia P. Hernández (CIC, "Comisión de Investigaciones Científicas de la Provincia de Buenos Aires") for the technical support. This work was supported by the National Agency of Promotion of Science of Argentina (ANPCyT, grants BID-PICT 2016-2798 and 2016-0739) and by the National University of La Plata (UNLP, grant 11/N852). This paper is dedicated in honor to Dr. Cleide Costa, in recognition of her contributions to the knowledge of Neotropical beetles.

\section{REFERENCES}

Costa, C.M.R.; Herrmann, G.; Martins, C.S.; Lins, L.V. \& Lamas, I.R. 1998. Biodiversidade em Minas Gerais: um atlas para sua conservação. Belo Horizonte, Fundação Biodiversitas.

del Río, M.G. \& Lanteri, A.A. 2011. Taxonomic revision of the genus Hadropus Schoenherr (Coleoptera: Curculionidae) with comments on infraspecific variation. Transactions of the American Entomological Society, 137(344): 307-315.

del Río, M.G. \& Lanteri, A.A. 2013. Taxonomic revision of the genus Stenocyphus Marshall (Coleoptera: Curculionidae) from Brazil. ZooKeys, 357: 29-43.

Farris, J.S. 1989. The retention index and the rescaled consistency index. Cladistics, 5: 417-419.

Felseinstein, J. 1985. Confidence limits on phylogenies: an approach using the Bootstrap. Evolution, 39: 783-791.

Goloboff, P.A. \& Catalano, S. 2016. TNT version 1.5, including a full implementation of phylogenetic morphometrics. Cladistics, 32(3): 221-238.

Goloboff, P.A. \& Farris, J.S. 2001. Methods for quick consensus estimation. Cladistics, 17: 26-34.

Kluge, A.G. \& Farris, J.S. 1969. Quantitative phyletics and the evolution of anurans. Systematic Zoology, 18: 1-32.

Lanteri, A.A. \& del Río, M.G. 2017. Phylogeny of the tribe Naupactini (Coleoptera: Curculionidae) based on morphological characters. Systematic Entomology, 42(2): 429-447.

Maddison, W.P. 1993. Missing data versus missing characters in phylogenetic analysis. Systematic Biology, 42: 576-581. 


\section{ADDITIONAL SUPPORTING INFORMATION}

Table S1. List of morphological characters and character states used for the phylogenetic analysis. The list and the figures called there are from Lanteri \& del Río (2017).

0. Squamose vestiture of the epistome: covered with scales similar in size, density and color to those of remainder of rostrum (0) (Fig. 4D); covered with scales smaller, sparser and usually of different color to those of remainder of rostrum (1) (Fig. 5E); lacking scales (2).

1. Vestiture of rostrum: setae on rostrum not forming semi-circles on sides of rostral sulcus (0); forming semi circles on sides of rostral sulcus (1) (Fig. 5C).

2. Vestiture of antennae: scape and funicle scarcely setose (0); scape squamose and funicle setose (1); scape and funicle squamose (2).

3. Vestiture, verticilate setae of antennal funicle (coarse, stiff setae around apex of antennal articles): setae light-colored (0); setae dark-colored (1).

4. Vestiture of scutellum: vestiture present, consisted on scales similar in color to those covering whole surface of elytra (0); vestiture present, consisted on white scales or seta-like scales (1) (Fig. 2B); vestiture absent (2).

5. Vestiture, dark maculae of seta-like scales on pronotum and elytra: dark maculae absent 0); dark maculae present (1) (Fig. 1E).

6. Scaly vestiture of elytra: uniformly present on whole surface of elytra (0) (Fig. 1B); forming longitudinal stripes or maculae and leaving glabrous areas (1) (Fig. 2A); elytra covered with seta-like scales (2) (Fig. 2E); elytra lacking scaly vestiture (3) (Fig. 2D).

7. Setose vestiture of elytra: absent (0) (Fig. 2D); setae short, recumbent to suberect, either fine or scale-like (1) (Fig. 3F); setae erect, long (about as long as width of interstria 2 near middle) (2) (Fig. 2E); setae erect, very long (longer than width of elytral interstriae 2 near middle) (3) (Fig. 3B).

8. Rostrum, expansion of apex in dorsal view (including borders of scrobes): rostrum expanded at apex, wider than frons (0) (Fig. 4A); rostrum not expanded at apex, equal to narrower than frons (1) (Fig. 5B).

9. Rostrum, curvature of dorso-lateral margins: dorso-lateral margins divergent towards apex (0) (Fig. 4B); margins subparallel to convergent towards apex (1) (Fig. 5B); margins curved and convergent towards apex in males and subparallel in females (2); margins curved and convergent towards apex in both sexes (3) (Fig. 4E).

10. Rostrum, curvature in profile view: rostrum with dorsal surface convex (0); rostrum with flat or depressed dorsal surface (1).

11. Rostrum, pair of lateral carinae: carinae indistinct (0) (Fig. 4F); carinae subparallel to convergent towards frons (1) (Fig. 5A); carinae divergent towards frons (2) (Fig. 5C).

12. Rostrum, borders of scrobes: not to slightly thickened (0) (Fig. 5F); strongly thickened (1) (Fig. 4B).

13. Rostrum, apex of scrobes in dorsal view: scrobes completely exposed from above (0) (Fig. 4A); scrobes slightly visible from above (1) (Fig. 4C).

14. Rostrum, scrobes in lateral view: slightly curved, posteriorly shallow and oriented towards eye (0); variously curved downwards towards ventral margin of eye (1) (Fig. 2E); strongly curved downward (almost 90 ) and directed ventrad of head (2) (Fig. 3E).

15. Rostrum, elevation of epistome: epistome not elevated relative to post-rostrum (0) (Fig. 5B); epistome elevated relative to post-rostrum (1) (Fig. 5D).

16. Rostrum, nasal plate sensu Vaurie, 1963: large, triangular, not descending, separated from epistome by a carina or elevated border (0) (Fig. 4B); narrow, V-shaped, descending, not separated from epistome by a carina or elevation (1) (Fig. 5A); very narrow, descending, not visible from above (2) (Fig. 4E).

17. Rostrum, presence of median groove: median groove absent along rostrum (0) (Fig. $4 \mathrm{~A}$ ); median groove always present along rostrum and frons (1) (Fig. 5E).

18. Rostrum, ventral side, position and extension of occipital sutures: occipital sutures well-developed, transversal (0); occipital sutures well-developed, oblique (1); occipital sutures reduce to indistinct (2) [See Franz, 2012, Fig. 30, char. 25.0, and Fig. 3, char. 25.1].

19. Rostrum, ventral side, hypostomal-labial sutures: ventral side without large triangular impression flanked by foveate hypostomal-labial sutures (0); with elongate triangular impression flanked by long, non-foveate hypostomal-labial sutures (1) [See Franz, 2012, Fig. 28, char. 24(0) and Fig. 29, char. 24(1)].

20. Mouthparts, setation of prementum: prementum lacking setae (0); prementum with various long setae (1).

21. Head, pair of arched grooves on sides of frons, around eyes: absent (0); present (1) (Fig. 5F).

22. Head, presence of preocular sulcus: preocular sulcus present (0) (Fig. 4C); preocular sulcus absent (1) (Fig. 4D).

23. Head, supraocular borders: not thickened (0); thickened (1) (Fig. 1F).

24. Head, position of eyes: eyes distinctly encroaching frons (0) (Fig. 4B); slightly encroaching frons (1) (Fig. 4D); entirely lateral (2) (Fig. 4F).

25. Head, shape of eyes: eyes oval, dorso-ventrally oriented (0) (Fig. 3E); eyes rounded to slightly oval and antero-posteriorly oriented (1) (Fig. 1E).

26. Head, postocular constriction: constriction slight to absent (0), constriction strong (1) (Fig. 5B).

27. Head, lateral view: gular angle very obtuse (0); slightly obtuse (1); almost right angled (2).

28. Antennae, cross-sectional shape of scape: scape cylindrical in cross section (0) (Fig. 5A); scape flat in cross section (1) (Fig. 4E).

29. Antennae, shape of scape: scape clavate (0) (Fig. 4D); scape capitate (1) (Fig. 5C); scape strongly clavate (2) (Fig. 4F); scape spatulate (3) (Fig. 4E).

30. Antennae, curvature of scape: scape not curved (0) (Fig. 5A); scape curved (1) (Fig. 5D).

31. Antennae, length of scape with respect to hind margin of eye or apex of pronotum: scape not reaching hind margin of eye (0) (Fig. 4C); reaching to slightly exceeding hind margin of eye (1) (Fig. 5D); reaching to slightly exceeding apex of pronotum (2) (Fig. 2F); largely exceeding apex of pronotum (3) (Fig. 4A).

32. Antennae, sexual dimorphism: scape width subequal in males and females (0); scape very broad in males and slender in females (1).

33. Antennae, length of funicular article 2 (antennomere 3) with respect to length of funicular article 1 (antennomere 2): funicular article 2 slighlty shorter to about as long as article 1 (0); funicular article 2 less than $2 \times$ longer than article 1 (1); funicular article 2, more than $2 \times$ longer than article 1 (2).

34. Antennae, length to width ratio of funicular article 3 (antennomere 4): funicular article 3 about as long as, to slightly longer than wide (0); distinctly elongate (more than $2 \times$ as long as wide) (1).

35. Antennae, length to width ratio of funicular article 7 (antennomere 8): funicular article 7 wider than long or as long as wide at apex (0); distinctly longer than wide at apex (1).

36. Antennae, length of funicular article 3 (antennomere 4) regarding funicular article 7 (antennomere 8): funicular article 3 longer than funicular article 7 (0); funicular article 3 as long as, to shorter than article 7 (1).

37. Antennae, shape of club: club oval, very short (length/width 2 to $2.25 \times$ ) (0); club oval, moderately short (length/width 2.26 to $3 \times$ ) (1); fusiform, very long (length/width more than $3 \times)(2)$. 
38. Pronotum, shape in dorsal view: subcylindrical (sides curved, maximum width about middle) (0) (Fig. 2D); slightly conical (sides divergent towards middle and subparallel on posterior half) (1) (Fig. 3B); strongly conical (sides strongly divergent from apex to base) (2) (Fig. 1B).

39. Pronotum, vibrissae on anterior margin: vibrissae absent (0); vibrissae present (1).

40. Pronotum, lateral tubercles at about middle of flanks: tubercles absent (0); tubercles present (1) (Fig. 1C).

41. Pronotum, median impression, longitudinal groove and/or elevation: disc of pronotum with fine, usually interrupted median groove (0); disc of pronotum with broad median groove (1) (Fig. 1C); disc of pronotum with median impression and groove (2) (Fig. 1A); disc of pronotum elevated along midline (3).

42. Pronotum, pair of lateral, longitudinal impressions: lateral impressions absent (0); lateral impressions present (1) (Fig. 1A).

43. Pronotum, pairs of short, oblique lateral sulci: lateral sulci absent (0); lateral sulci present (1) (Fig. 3A).

44. Pronotum, curvature of disc in lateral view: flat to slightly convex (0); strongly convex (1) (Fig. 2F).

45. Pronotum, postero-lateral angles (females): postero-lateral angles not projecting (0) (Fig. 2C); postero-lateral angles projecting (1); postero-lateral angles projecting in a tubercle (2) (Fig. 2B).

46. Pronotum, constriction and thickness of margins: anterior and posterior margins not constricted, not to slightly thickened (0); posterior margin constricted and thickened (1) (Fig. 2B); anterior and posterior margins strongly constricted and thickened (2) (Fig. 3D).

47. Pronotum, outline of base: base straight to slightly bisinuate (0); base strongly bisinuate (1) (Fig. 3A).

48. Pronotum, sculpture in dorsolateral profile: disc of pronotum smooth to slightly punctuate (0) (Fig. 2D); disc of pronotum with irregular foveae or large punctures (1) (Fig. 3A); disc of pronotum granulose (2) (Fig. 3C); disc of pronotum striate (3).

49. Scutellum, shape: scutellum quadrate to rounded (0); scutellum triangular (1); scutellum reduce to indistinct (2).

50. Elytra, outline of base: base of elytra straight (0) (Fig. 2C); base of elytra slightly bisinuate (1) (Fig. 1C); base of elytra strongly bisinuate (2) (Fig. 3A).

51. Elytra, development of humeri: humeri well-developed (0) (Fig. 2A); humeri reduced (1) (Fig. 3C); humeri absent (2) (Fig. 2C).

52. Elytra, presence of humeral tubercle: humeri lacking tubercle (0); humeri projecting in a small anteriorly directed tubercle (1) (Fig. 1D); humeri projecting in a postero-laterally directed tubercle (2) (Fig. 1C); humeri projecting in a long laterally directed tubercle (3) (Fig. 2D).

53. Elytra, presence of hump: elytra not humped (not ascending towards declivity) (0) (Fig. 2E); elytral disc humped (ascending towards declivity) (1) (Fig. 1E).

54. Elytra, height in lateral view: elytra very high (0) (Fig. 3E); elytra moderately high (1) (Fig. 2E); elytra flat (2) (Fig. 2F).

55. Elytra, development of subapical calli: subapical calli well-developed (0) (Fig. 3A); subapical calli slightly developed (Fig. 2B) (1); subapical calli indistinct (2) (Fig. 2C).

56. Elytra, apical declivity: apical declivity abrupt (0) (Fig. 3F); apical declivity slight to moderate (1) (Fig. 2F).

57. Elytra, presence of tubercles on disc and declivity: elytra lacking tubercles (0); elytra with tubercles (1) (Fig. 1F).

58. Elytra, outline of apex: apex entire (0); apex bifid (1) (Fig. 3D).

59. Elytra, curvature of intervals (= interstriae): intervals flat (0) (Fig. 1B); intervals slightly convex (1); uneven intervals (except suture) strongly convex, even intervals flat (2) (Fig. 1A); all intervals strongly convex (3) (Fig. 2D).

60. Elytra, presence of supernumerary striae: supernumerary striae absent (0); supernumerary striae (more than 10 striae in each elytrum) present (1) (Fig. 1A).

61. Elytra, proximity of striae $9^{\circ}$ and $10^{\circ}$ : elytra with striae $9^{\circ}$ and $10^{\circ}$ fused along posterior $\otimes(0)$; slightly closer to each other along posterior $\bigotimes(1)$; well separated along their extension (2).

62. Elytra, size of punctures of striae: punctures large (about as wide as, to slightly wider than intervals) (0) (Fig. 3A); punctures medium-sized to small (narrower than intervals) (1) (Fig. 1A); punctures very small and close to each other (2) (Fig. 1B). (The ratio width of interval/width of puncture was measured on intervals 2 to 5 , about middle length of elytra).

63. Metathoracic wings, development: wings well-developed (0); wings reduced to absent (1).

64. Legs, separation of front coxae (females): front coxae contiguous (0); front coxae separated from each other (1).

65. Legs, distance of front coxae regarding margins of prosternum: front coxae closer to posterior than to anterior margin of prosternum (0); equally separated from anterior and posterior margins of prosternum or slightly closer to anterior margin (1); twice as close to anterior margin than to posterior margin of prosternum (2).

66. Legs, width of front femora relative to width of hind tibiae (females): hind femora wider than front femora (0) (Fig. $3 \mathrm{E}$ ); front femora about as wide as, to slightly wider (up to 1.25x) than hind femora (1) (Fig. 1E); front femora wider than hind femora (more than 1.25x and less than 1.50x) (2); front femora strongly widened (1.50× or more, as wide as hind femora) (3) (Fig. 3D).

67. Legs, presence of denticles in front femora: denticles of femora absent (0); front femora with one to three small denticles on inner side (1); all femora with one large denticle (2).

68. Legs, size of mucro of front tibiae: mucro indistinct (0); mucro small to medium-sized (less than half, or about as long as half width of tibial apex) (1); mucro large (about as long as width of tibial apex) (2).

69. Legs, front tibiae: without prominence opposite to mucro (0); with prominence opposite to mucro (1).

70. Legs, presence of row of denticles on inner margin of tibiae: denticles absent to minute (0); denticles present in front tibiae only (1); denticles present in the three pairs of tibiae (2).

71. Legs, corbel at metatibial apex: corbel broad, squamose ( 2 to $2.5 \times$ as long as wide) (0); corbel narrow squamose or setose (more than $3 \times$ as long as wide) (1); corbel lacking (= metatibial apex simple) (2).

72. Legs, length of dorsal comb relative to length of distal comb of hind tibiae: dorsal comb shorter than distal comb (0); dorsal comb about as long as distal comb (1); dorsal comb longer than dorsal comb (2). [See Buchanan, 1939, pag. 7, Figs. 2J, 2B, 2I].

73. Legs, length relative to width of tarsite 2 of hind tibiae: tarsite 2 wider than long (0); tarsite 2 about as long as wide (1); tarsite 2 longer than wide (2).

74. Venter, width of intercoxal portion of first abdominal ventrite: intercoxal portion about as wide as, to slightly narrower than space of hind coxae (0); intercoxal portion distinctly wider than space of hind coxae (1).

75. Venter, presence of impression on ventrites 1 and 2 (females): ventrites 1 and 2 flat, lacking impression (0); ventrites 1 and 2 with a median impression (1).

76. Venter, length of ventrite 2 relative to length of ventrites $3+4$, measured along midline: ventrite 2 twice as long as ventrites $3+4(0)$; slightly longer than ventrites $3+4$ (1); about as long as ventrites $3+4$, to slightly shorter (2). 
77. Venter, length of ventrite 1 regarding relative to ventrite 2 , measured along midline: ventrite 1 distinctly longer than ventrite $2(0)$; ventrite 1 about as long as ventrite 2 (1).

78. Female terminalia, shape of sternite VIII: subtriangular (0) (Fig. 6A); subrhomboidal, very elongate (1) (Fig. 6B); subrhomboidal, slightly elongate (2) (Fig. 6C); rounded to suboval (3) (Fig. 6D); subcordiform (4) (Fig. 6E); subpentagonal (= shovel-shaped) (5) (Fig. 6F).

79. Female terminalia, apical margin of sternite VIII: sternite VIII entire (0); sternite VIII divided (1); sternite VIII divided and projected in a pair of denticles (2) (Fig. 6G).

80. Female terminalia, length of apodeme of sternite VIII: apodeme less than $2 \times$ as long as plate (0) (Fig. 6A); apodeme equally long or more than $2 \times$ as long as plate (1) (Fig. 6D).

81. Female terminalia, length of ovipositor (distal plus dorsal gonocoxites): ovipositor about $\bigotimes$ of abdominal length (0); ovipositor about half length of abdomen (1); ovipositor about 3/4 of abdominal length (2); about as long as, to slightly longer than abdomen (3).

82. Female terminalia, presence of baculi along ovipositor: baculi absent (0) (Fig. 7A); baculi present (1) (Fig. 7B).

83. Female terminalia, presence of rows of setae along each side of baculi: ovipositor lacking rows of setae (0); ovipositor with rows of setae (1) (Fig. 7B).

84. Female terminalia, shape and degree of sclerotization of distal gonocoxites: distal gonocoxites slightly to moderately sclerotized, not projected (0) (Fig. 7B); strongly sclerotized and projected in a short nail-shaped piece (1) (Fig. 7C); strongly sclerotized and strongly projected in a long nail- shaped piece (2) (Fig. 7D).

85. Female terminalia, presence of styli: styli present, visible from ventral side (0) (Fig. 7B); styli present, hidden by distal gonocoxites from ventral side (1) (Fig. 7E); styli absent (2) (Fig. 7C).

86. Spermatheca, width of spermathecal duct: spermathecal duct fine (0) (Fig. 8D); spermathecal duct wide (1) (Fig. 8B).

87. Spermatheca, undulation of spermathecal duct: spermathecal duct straight to slightly undulate (Fig. 8A) (0); spermathecal duct curled (1) (Fig. 8B).

88. Spermatheca, sclerotization of spermathecal duct: spermathecal duct slightly sclerotized (0); spermathecal duct as sclerotized as spermatheca (1).

89. Spermatheca, shape of corpus: corpus subcylindrical, slender (about as wide as base of cornu) (0) (Fig. 8B); corpus broadly subcylindrical (1) (Fig. 8C); corpus amphora-shaped (2) (Fig. 8D); corpus subspherical (3) (Fig. 8F).

90. Spermatheca, position of ramus: ramus inserted laterally on corpus of spermatheca (0) (Fig. 8G); ramus inserted on proximal extreme of corpus, same as collum (1) (Fig. 8E).

91. Spermatheca, development of ramus: ramus distinct (0) (Fig. 8G); ramus indistinct (1) (Fig. 8A).

92. Spermatheca, shape and length of collum (= nodulus): collum indistinct (0) (Fig. 8D); conical, short (1) (Fig. 8A); collum conical, moderately long (2) (Fig. 8B); collum subcylindrical, long, curved in opposite direction regarding apex of cornu (3) (Fig. 8G); collum subcylindrical, long, extended in same direction of corpus (4) (Fig. 8H).

93. Spermatheca, basal prominence of collum: basal prominence absent (0); basal prominence present (1) (Fig. 8B).

94. Spermatheca, length of cornu: cornu long (longer than corpus) (0) (Fig. 8G); medium length (about as long as corpus) (1) (Fig. 8C); short (shorter than corpus) (2) (Fig. 8D).

95. Aedeagus, length of apodemes (= temones): apodemes about as long as median lobe (0) (Fig. 9G); apodemes about half length of median lobe (1) (Fig. 9F); apodemes twice as long as median lobe (2).

96. Aedeagus, shape of apex of median lobe: apex acute to slightly acute (0) (Fig. 9A); apex truncate (1) (Fig. 9B); apex rounded (Ericydeus type) (2) (Fig. 9C); apex arrow- shaped (3) (Fig. 9D); apex horn- shaped (4) (Fig. 9E).

97. Aedeagus, presence of setae on apex: setae absent (0) (Fig. 9F); setae present around ostium (1) (Fig. 9G); setae present around ostium and on ventral surface (2) (Fig. 9H).

98. Aedeagus, presence of flagellum: flagellum absent (0); flagellum present (1).

99. Aedeagus, sclerites of the internal sac: sclerites absent or not Cyrtomon type (0); sclerites consisted on a pair of lateral struts on each side of a pyriform piece connected with ejaculatory duct = Cyrtomon type (1) (Fig. 9l). 


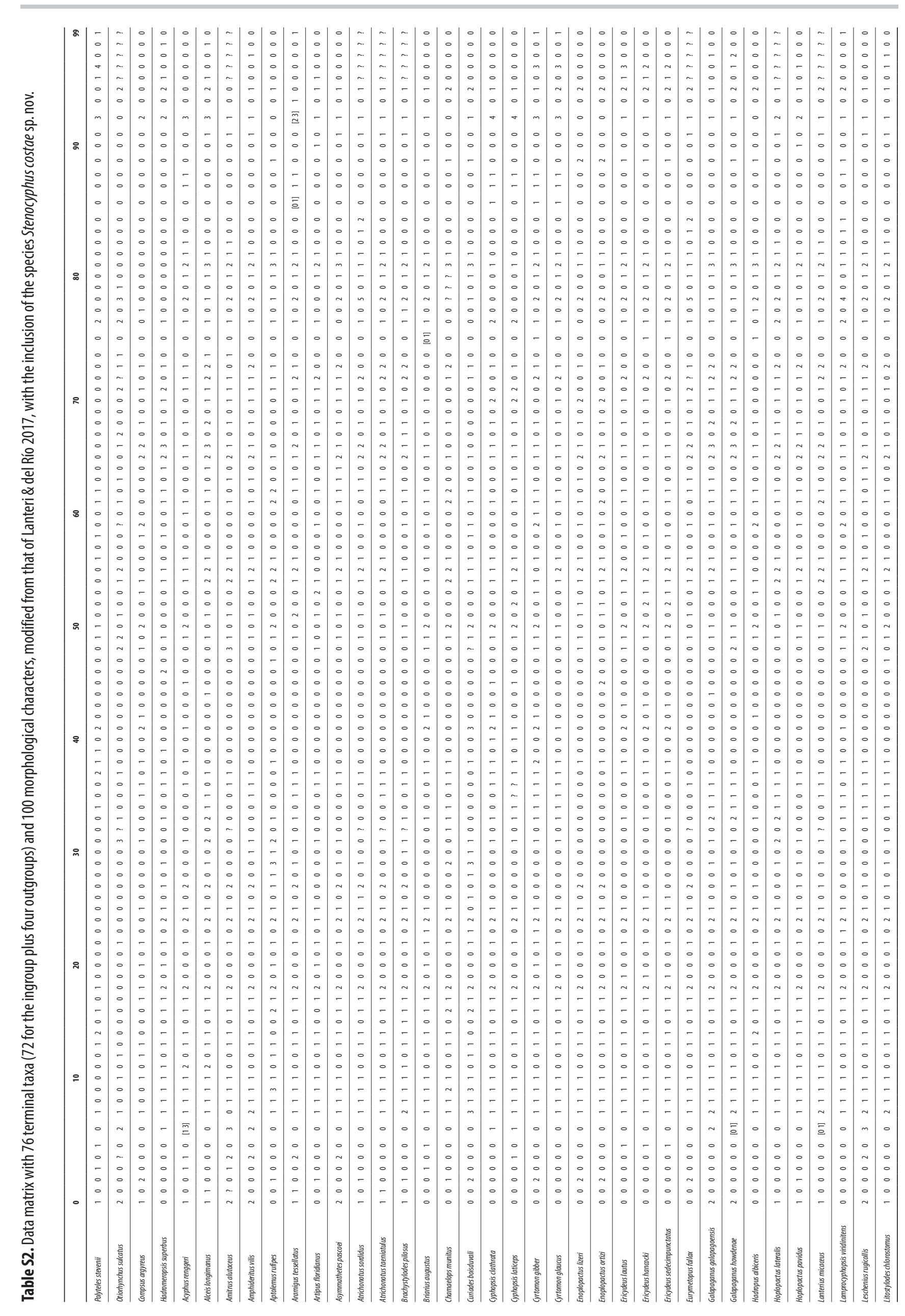




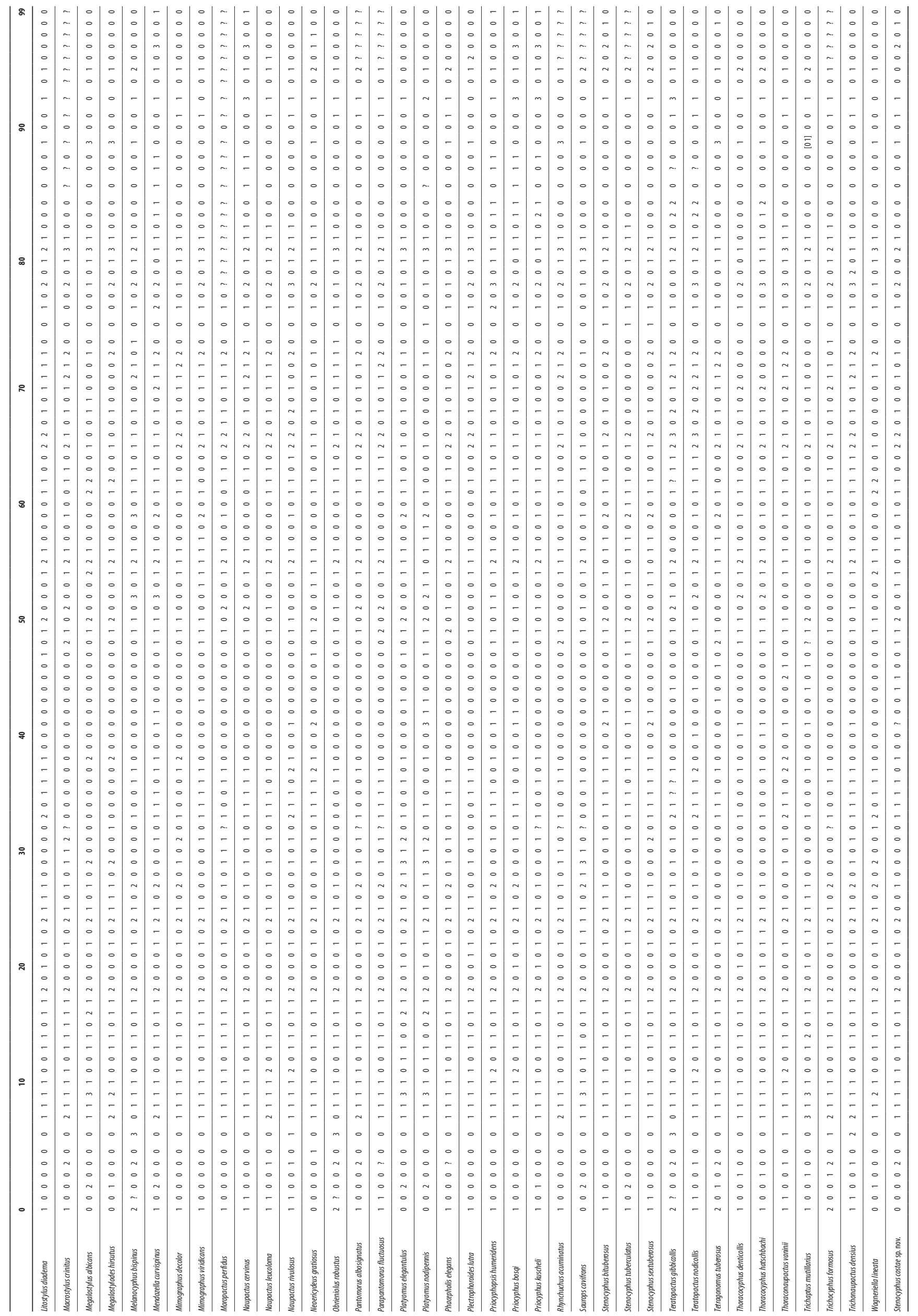

